\title{
Optimal approach to obtaining mucosal biopsies for assessment of eosinophilic esophagitis and lymphocytic esophagitis
}

\begin{abstract}
Eosinophilic esophagitis (EoE) and lymphocytic esophagitis (LyE) are inflammatory disorders of esophagus characterized clinically by dysphagia or food impaction. Endoscopical findings like esophageal rings, exudates, furrows and strictures might indicate the presence of these conditions, however, these findings have also been described in other esophageal disorders and endoscopic appearance may be normal in up to $25-30 \%$ patients. Inflammation may display a patchy distribution and multiple biopsies from different parts during an endoscopy procedure are required to ensure diagnostic accuracy. Determination of the most appropriate site of the esophagus to obtain a biopsy specimen for diagnosis of EoE or LyE is considered critical. This article highlights issues related to optimal approach to obtaining mucosal biopsies for assessment of EoE and LyE.
\end{abstract}

Keywords: Eosinophilic esophagitis, Lymphocytic esophagitis, Esophageal biopsies
Volume 5 Issue 3 - 2016

\author{
Aldona Dlugosz \\ Department of Medicine Huddinge and Center for Digestive \\ Diseases, Karolinska Institutet and Karolinska University \\ Hospital, Sweden \\ Correspondence: Aldona Dlugosz, Department of Medicine \\ Huddinge Center for Digestive Diseases, Karolinska Institute \\ and Karolinska University Hospital, SE-14186, Stockholm, \\ Sweden, Tel +468585823 43, Fax +468585823 35, \\ Email aldona.dlugosz@karolinska.se
}

Received: July 14,2016 | Published: September 08, 2016
Abbreviations: EoE; Eosinophilic Esophagitis, HPF; High Power Field, IPCL; Intrapapillary Capillary Loops, LyE; Lymphocytic Esophagitis, NBI-ME; Narrow-Band Imaging Magnifying Endoscopy

\section{Introduction and discussion}

EoE is defined as a chronic, immune/antigen-mediated, esophageal disease characterized clinically by symptoms related to esophageal dysfunction and histologically by an eosinophil-predominant inflammation ( $\geq 15$ eosinophils per high power field (hpf)) in the esophageal mucosa. ${ }^{1}$ Endoscopic abnormalities in patients with EoE include esophageal rings, strictures, narrow-caliber esophagus, linear furrows, white plaques or exudates and edema. ${ }^{2}$ However, there are no pathognomonic signs for EoE since these endoscopic findings have also been described in other esophageal disorders. Moreover, endoscopic appearances may be normal in $10-25 \%$ of EoE patients. ${ }^{3}$

Lymphocytic esophagitis (LyE) was first described as an independent entity in 2006 by Rubio et al. ${ }^{4}$ LyE patients clinically present with dysphagia, abdominal pain, heartburn and nausea. ${ }^{5}$. The diagnosis of LyE is considered when more than 40 intraepithelial lymphocytes/hpf are present, and none or only occasional CD15+ intraepithelial granulocytes. ${ }^{4,6}$ Endoscopic features of LyE can be similar to EoE including esophageal rings, furrows, exudates, narrow lumen or stenosis but in one-third of patients the esophageal mucosa appears macroscopically normal. ${ }^{5}$

By consensus, an eosinophilic infiltrate of $\geq 15$ eosinophils /hpf suggests the diagnosis of EoE. ${ }^{1}$ however the distribution of esophageal eosinophilia is often patchy. ${ }^{7,8}$ There are no studies describing the distribution of lymphocytic infiltration in LyE. Multiple biopsy samples from different parts during an endoscopy procedure are required to ensure diagnostic accuracy. ${ }^{7}$ However, multiple biopsies of the esophagus are not easily carried out during an endoscopy examination in clinical practice. Therefore, determination of the most appropriate site of the esophagus to obtain a biopsy specimen for diagnosis of EoE or LyE is considered critical for gastroenterologists. ${ }^{9}$
Patients with EoE often demonstrate eosinophilic infiltration in both distal and proximal biopsies, although proximal biopsies vary in eosinophil density on a patient-to-patient basis. ${ }^{10}$ Gonsalves et al. ${ }^{10}$ found that, when the threshold of $\geq 15$ eosinophils /hpf was applied, evaluation of a single biopsy specimen yielded a sensitivity rate of only $55 \%$ for detection of EoE, compared to $100 \%$ when five biopsies were obtained from the proximal, middle and distal esophagus. ${ }^{10}$ Nielsen et al. ${ }^{11}$ demonstrated that four to six biopsy fragments from distal and proximal esophagus should be submitted to optimize the chances of achieving the morphologic criteria for a diagnosis of EoE, and that the field is not increased beyond six biopsy fragments.

An endoscopic feature suggesting EoE (rings, furrows, exudates, narrow lumen and stenosis) does not always represent esophageal eosinophilia although it reminds endoscopist of the presence of EoE. ${ }^{12}$ Pooled analysis showed that at least one such feature was detected by endoscopy in up to $93 \%$ of patients with EoE. ${ }^{13}$

Endoscopic appearance and location dictate diagnostic yield of biopsies in EoE. However, there are some discrepancies about the suitable biopsy site for detection of eosinophilia. Adachi et al. ${ }^{9}$ reported severe exudates as a significant factor related to a positive finding of eosinophilic infiltration. Salek et al. ${ }^{14}$ reported also higher eosinophil counts in biopsies obtained from exudates and furrows although rings alone without associated furrows and exudates did not demonstrate elevated eosinophil counts. In contrary, in a study by Hori et al. ${ }^{12}$ diagnostic utility of linear furrows or rings was superior to that of white exudates.

Tanaka et al. ${ }^{15}$ recently described narrow-band imaging magnifying endoscopy (NBI-ME) criteria for EoE and LyE. Three abnormal NBI-features were identified:

i. Beige color of the mucosa,

ii. Increased and dot-shaped congested intrapapillary capillary loops (IPCL) and

iii. Invisibility of submucosal vessels. The presence of at least one of the above findings was reported in $100 \%$ patients with EoE and $91 \%$ of patients with LyE. 
In author's own experience NBI-ME criteria are useful to determine the site of inflammation. To this date we have no established guidelines regarding biopsy protocol in LyE. However, the fact that the endoscopic appearances may be normal in up to $25 \%$ of EoE patients and $30 \%$ of LyE patients supports the recommendation for biopsy acquisition in all patients with dysphagia and/or food bolus impaction.

\section{Conclusion}

Esophageal biopsies from the distal, middle and proximal esophagus with assessment of both eosinophils and lymphocytes should be obtained during endoscopy for any patient with a history of dysphagia or food bolus impaction. Endoscopists should probably focus their biopsies towards areas of exudates and furrows, as these areas are likely to represent increased eosinophil infiltrate. NBIME may be helpful to identify the site of inflammation and allows targeted biopsies in both EoE and LyE. Additionally, the samples from duodenum and stomach should also be obtained to exclude other causes of eosinophilic and/or lymphocytic infiltration such as eosinophilic gastritis or gastroenteritis, Crohn's or celiac disease.

\section{Financial support}

Author was supported by the Stockholm County Council (clinical post doctorial appointment).

\section{References}

1. Liacouras CA, Furuta GT, Hirano I, etal.Eosinophilic esophagitis:updated consensus recommendations for children and adults. $J$ Allergy Clin Immunol. 2011;128(1):3-20 e26.

2. Dellon E. Eosinophilic esophagitis. Gastroenterol Clin North Am. 2013;42(1):133-153.

3. Dellon ES, Gonsalves N, Hirano I, et al.ACG clinical guideline:Evidenced based approach to the diagnosis and management of esophageal eosinophilia and eosinophilic esophagitis (EoE). Am J Gastroenterol. 2013;108(5):679-692.
4. Rubio CA, Sjodahl K, Lagergren J. Lymphocytic esophagitis:a histologic subset of chronic esophagitis. Am J Clin Pathol. 2006;125(3):432-437.

5. Cohen S, SaxenaA, WaljeeAK, et al.Lymphocytic esophagitis:a diagnosis of increasing frequency. J Clin Gastroenterol. 2012;46(10):828-832.

6. Akiyama J, Bertele A, Brock C, et al. Benign and precursor lesions in the esophagus. Ann N Y Acad Sci. 2014;1325:226-241.

7. Dellon ES, Speck O, Woodward K, et al. Distribution and variability of esophageal eosinophilia in patients undergoing upper endoscopy. Mod Pathol. 2015;28(3):383-390.

8. Saffari H, Peterson KA, Fang JC, et al. Patchy eosinophil distributions in an esophagectomy specimen from a patient with eosinophilic esophagitis:Implications for endoscopic biopsy. J Allergy Clin Immunol. 2012;130(3):798-800.

9. Adachi K, Mishiro T, Tanaka S, et al. Suitable biopsy site for detection of esophageal eosinophilia in eosinophilic esophagitis suspected cases. Dig Endosc. 2016;28(2):139-144.

10. Gonsalves N, Policarpio-Nicolas M, Zhang Q, et al. Histopathologic variability and endoscopic correlates in adults with eosinophilic esophagitis. Gastrointest Endosc. 2006;64(3):313-319.

11. Nielsen JA, Lager DJ, Lewin M, etal. The optimal number of biopsy fragments to establish a morphologic diagnosis of eosinophilic esophagitis. Am J Gastroenterol. 2014;109(4):515-520.

12. Hori K, Watari J, Fukui H, et al. Do endoscopic features suggesting eosinophilic esophagitis represent histological eosinophilia? Dig Endosc. 2014;26(2):156-163.

13. Kim HP, Vance RB, Shaheen NJ, et al. The prevalence and diagnostic utility of endoscopic features of eosinophilic esophagitis:a metaanalysis. Clin Gastroenterol Hepatol. 2012;10(9):988-996.

14. Salek J, Clayton F, Vinson L, et al. Endoscopic appearance and location dictate diagnostic yield of biopsies in eosinophilic oesophagitis. Aliment Pharmacol Ther. 2015;41(12):1288-1295.

15. Tanaka K, Rubio CA, Dlugosz A, et al. Narrow-band imaging magnifying endoscopy in adult patients with eosinophilic esophagitis/ esophageal eosinophilia and lymphocytic esophagitis. Gastrointest Endosc. 2013;78(4):659-664. 